\section{Large observational study on risks predicting emergency department return visits and associated disposition deviations}

\author{
Charles Huggins, Richard D. Robinson, Heidi Knowles, Jennalee Cizenski, \\ Rosalia Mbugua, Jessica Laureano-Phillips, Chet D. Schrader, \\ Nestor R. Zenarosa, Hao Wang
}

Department of Emergency Medicine, Integrative Emergency Services, John Peter Smith Health Network, Fort Worth, TX, USA

Objective A common emergency department (ED) patient care outcome metric is 72-hour ED return visits (EDRVs). Risks predictive of EDRV vary in different studies. However, risk differences associated with related versus unrelated EDRV and subsequent EDRV disposition deviations (EDRVDD) are rarely addressed. We aim to compare the potential risk patterns predictive of related and unrelated EDRV and further determine those potential risks predictive of EDRVDD.

Methods We conducted a large retrospective observational study from September 1, 2015 through June 30, 2016. ED Patient demographic characteristics and clinical metrics were compared among patients of 1) related; 2) unrelated; and 3) no EDRVs. EDRVDD was defined as obvious disposition differences between initial $E D$ visit and return visits. A multivariate multinomial logistic regression was performed to determine the independent risks predictive of EDRV and EDRVDD after adjusting for all confounders.

Results A total of 63,990 patients were enrolled; 4.65\% were considered related EDRV, and $1.80 \%$ were unrelated. The top risks predictive of EDRV were homeless, patient left without being seen, eloped, or left against medical advice. The top risks predictive of EDRVDD were geriatric and whether patients had primary care physicians regardless as to whether patient returns were related or unrelated to their initial ED visits.

Conclusion Over $6 \%$ of patients experienced ED return visits within 72 hours. Though risks predicting such revisits were multifactorial, similar risks were identified not only for ED return visits, but also for return ED visit disposition deviations.

Keywords Emergency service, hospital; Patient outcome assessment; Return visit
eISSN: 2383-4625

Received: 8 April 2018

Revised: 28 June 2018

Accepted: 4 July 2018

Correspondence to: Hao Wang Department of Emergency Medicine, Integrative Emergency Services, John Peter Smith Health Network, $1500 \mathrm{~S}$. Main St., Fort Worth, TX 76104, USA E-mail: hwang01@jpshealth.org ORCID

http://orcid.org/0000-0002-5105-0951

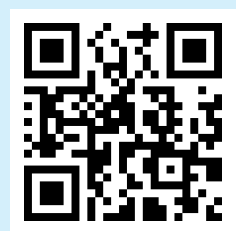

How to cite this article:

Huggins $\mathrm{C}$, Robinson RD, Knowles $\mathrm{H}$, Cizenski J, Mbugua R, Laureano-Phillips J, Schrader CD, Zenarosa NR, Wang H. Large observation study on risks predicting emergency department return visits and associated disposition deviations. Clin Exp Emerg Med 2019;6(2):144-151.

This is an Open Access article distributed under the terms of the Creative Commons Attribution Non-Commercial License (http:// creativecommons.org/licenses/by-nc/4.0/). 


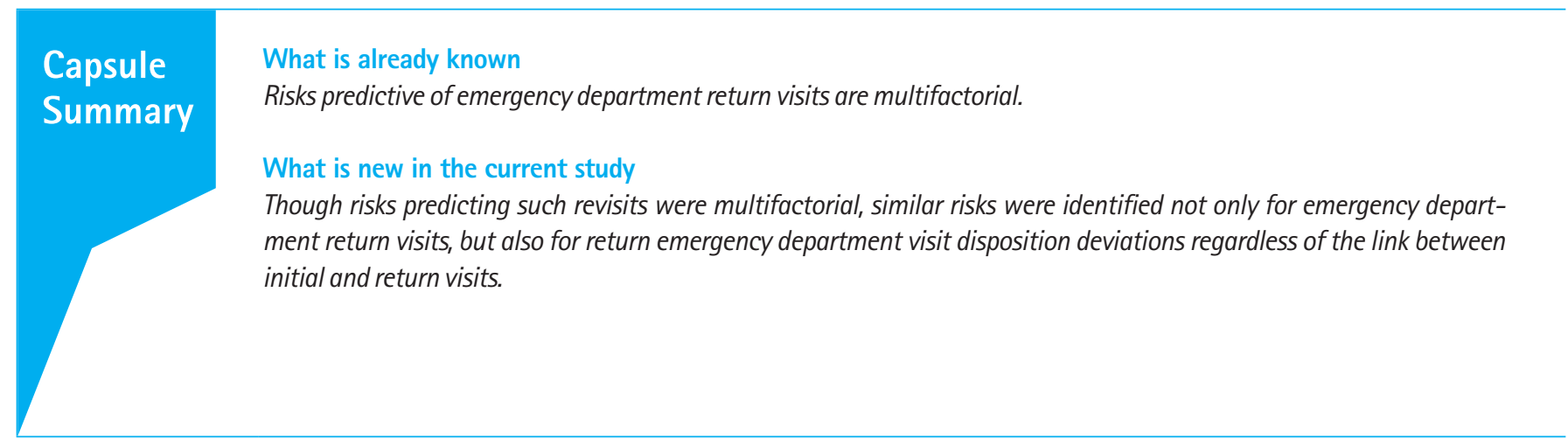

\section{INTRODUCTION}

Emergency department return visits (EDRV) are considered a measure of patient safety. Higher EDRV rates indicate negative patient care outcomes. ${ }^{1-3}$ Some studies focused on EDRV within 72 hours report increased morbidity and hospital readmission among the revisit group ${ }^{4,5}$ while others reported similar disease patterns and hospital admissions in comparison to patients with no 72 hours EDRV. ${ }^{6,7}$

To better understand patients experiencing emergency department (ED) returns, potential risks predictive of such returns have been extensively studied. ${ }^{8-10}$ Of note, a variety of potential risks were identified among the different studies. Three common risk categories identified in these studies are illness, patient, and provider/system related factors. ${ }^{11}$ IIIness-related risks focus on different diseases or complaints posing higher risk of EDRV. Examples are abdominal pain, cancer, alcohol/drug abuse, psychiatric/chronic disease conditions, etc. ${ }^{9,10,12}$ These high-risk potential EDRV are typically thought to be related to initial ED visits. However, direct supporting evidence is generally lacking. Patient-related risks mainly address patient specific characteristics (age, sex, insurance type, etc. ${ }^{13-16}$ and provider/system-related risks identify healthcare systems issues (provider characteristics, completion of discharge instructions, ED crowding, etc.). ${ }^{17-19}$ Previous studies focusing on the latter two types of risks neither differentiate whether EDRV are related to the initial ED visits nor do they specifically identify risks predictive of a relationship between EDRV and the initial visit. Uncertainty remains as to whether EDRV truly alters patient disposition and potential risks predictive of such disposition deviations from initial ED visits.

Simply determining risks predictive of EDRV without knowing whether these visits are related to the initial visit is insufficient in terms of establishing the predictive value of future interventions unless these risks are identical. Additionally, interventions might not be sufficient without knowing any potential significant disposition deviations that occur among ED return visits. Therefore, we aim to determine 1) the potential risks predictive of 72 hours related EDRV among all discharged ED patients and 2) the potential risks predictive of ED disposition deviations among all EDRV patients.

\section{METHODS}

\section{Study design and setting}

This is a retrospective, observational, single-center study. All data were retrieved from electronic health records visits covering the period September 1, 2015 through June 30, 2016. The study setting is an urban ED with annual patient visits $>120,000$. The institutional review board of John Peter Smith Health Network approved this study (010713.004ex). Due to retrospective chart review with no more than minimal risk to the subjects and the research could not practicably be done without a waiver of consent, this study was approved with the waiver of the written informed consent.

\section{Participants}

We included all patients presenting to the study hospital ED that 1) were discharged, 2) left without being seen (LWBS), 3) left against medical advice (AMA), and 4) eloped during the initial ED visits. Patients who visited the ED multiple times during the study period were considered as different patient encounters. We excluded 1) patients that expired at the initial ED visits, 2) patients admitted to hospital during the initial ED visits, 3) patients transferred to other facilities during initial ED visits, and 4) prisoners.

\section{Variables}

Patients who visited the study ED within 72 hours from the index ED visits were considered EDRV. Those who did not visit the study 
ED within 72 hours from the index visits were considered the noreturn group. Two individual ED physicians, blinded to the study's purpose, reviewed enrolled patient charts to determine whether EDRV were related or unrelated to the initial ED visits. To resolve discrepancies, a second round of reviews were rendered at least 30 days apart from the previous review. Repeated reviews occurred until a strong level of interrater agreement was reached.

Potential independent risks predictive of ED returns were divided into three categories: patient-related risks, system/providerrelated risks, and illness-related risks. Potential patient-related risks included age, sex, race/ethnicity, mode of arrival, insurance type, and homeless status. Mode of arrival was divided into three categories: 1) healthcare assisted transportation (ambulance ground transportation, flight, and hospital assisted transportation); 2) private transportation (private car or taxi); and 3) public/ other transportation (public vehicle, ambulatory, and wheelchair). Insurance type was divided into three categories: 1) government/ charity (Medicaid, Medicare, and other national/local charity insurance plans); 2) private commercial insurance; and 3) no insurance. Potential system/provider-related risks included initial total ED length of stay, initial ED disposition, and whether patients had their primary care physician assigned upon initial ED visit. Initial ED dispositions were further divided into two categories, including discharge and disposition with uncertainty, a combination of eloped, AMA, and LWBS either before or after triage completion. Potential illness-related risks included level of acuity as determined by the 5-point Emergency Severity Index, history of alcohol or substance abuse, psychiatric conditions, acute injury, and whether patients had discharge medications prescribed during the initial ED visits. Psychiatric conditions included patients with history of depression, anxiety, mania, schizophrenia, schizoaffective disorder, and bipolar disorder. Acute injury was defined as patients presenting to the ED with acute traumatic injury including, but not limited to, any type of trauma, stab wound, gunshot wound, laceration, and fracture/dislocation.

\section{Outcome measurements}

EDRV was categorized as related or unrelated EDRV. The primary outcome was to determine the risks predictive of related 72-hour EDRVs. The secondary outcome was to identify significant EDRV disposition deviation (EDRVDD) and further determine risks predictive of EDRVDD. Significant EDRVDD was defined as an obvious disposition difference between initial ED visit and return visit. Such disposition deviations included hospital admissions, hospital observation, patient expiration during EDRV, or operation room admission. Non-significant ED disposition deviation was defined as patients discharged during return visits. Uncertain ED disposi- tion deviation included patients that eloped, left AMA, LWBS, and those transferred to other services or facilities during EDRV.

\section{Data sources}

All data, except for related versus unrelated EDRV determination, were retrieved by persons from the hospital's information technology department who were blinded to the study's outcomes. To assess internal validation, twenty random samples were selected at three separate phases. As this is an epidemiological observational study, sample size estimation is not considered necessary per study design.

\section{Study protocol}

After individual physicians rendered intensive chart reviews, interrater variability was tested to determine consistency with respect to determination of 72 hours EDRV. Patients were initially divided into three groups: 1) patients with no EDRV, 2) patients with 72 hours related EDRV, and 3) patients with 72 hours unrelated EDRV. Potential risk variables were compared among the three groups. Risks predictive of patient 72 hours EDRV (both related and unrelated) were analyzed. Additionally, among all EDRV patients, those with significant EDRVDD were analyzed separately. Risks predictive of significant EDRVDD were compared between related and unrelated EDRV patients.

\section{Data analysis}

The kappa test was used for interrater variability analysis with $\kappa$ $>0.8$ indicating a strong level of agreement. The analysis of variance was used for continuous data comparisons among the three different groups and the Pearson chi-square test was used for categorical data comparison. We used multivariate multinomial logistic regression models to examine the relationship between the predictors and the outcomes of interest. ${ }^{20}$ We classified our primary outcome into three categories: no return, related return, and unrelated return. Predictors included age, sex, race/ethnicity, mode of arrival, primary care provider assignment, level of acuity, insurance type, homelessness, history of alcohol abuse, history of substance abuse, history of psychiatric conditions, ED length of stay, ED medication prescription upon discharge, and whether patients had acute injuries. The secondary outcome was classified into three categories: no disposition deviation, uncertain disposition deviation, and significant disposition deviation. All predictors included in the primary outcome analysis were included in this secondary outcome model prediction with the addition of two variables (return mode of arrival and return triage level of acuity). Diagnostic tests for collinearity and potential variable interactions were performed among predictor variables. Our final model 
Table 1. Study patient general characteristics

\begin{tabular}{|c|c|c|c|}
\hline Characteristics & No EDRV $(n=59,860,93.55 \%)$ & Related EDRV $(n=2,976,4.65 \%)$ & Unrelated EDRV $(n=1,154,1.80 \%)$ \\
\hline \multicolumn{4}{|l|}{ Initial ED visits } \\
\hline \multicolumn{4}{|l|}{ Age $(y r)$} \\
\hline Less than 65 & $56,360(94.6)$ & $2,748(92.7)$ & $1,072(93.1)$ \\
\hline 65 or older & $3,233(5.4)$ & $217(7.3)$ & $79(6.9)$ \\
\hline \multicolumn{4}{|l|}{ Sex } \\
\hline Female & $31,645(53)$ & $1,313(44)$ & 507 (44) \\
\hline Male & $27,945(47)$ & $1,652(56)$ & $644(56)$ \\
\hline \multicolumn{4}{|l|}{ Race } \\
\hline White & $21,567(36)$ & $1,312(44)$ & $508(44)$ \\
\hline Black & $20,617(34)$ & $1,080(36)$ & $443(38)$ \\
\hline Others ${ }^{a}$ & $17,676(30)$ & $584(20)$ & $203(18)$ \\
\hline \multicolumn{4}{|l|}{ Ethnicity } \\
\hline Hispanic & $16,641(28)$ & $579(20)$ & $209(18)$ \\
\hline Non-Hispanic & $42,292(72)$ & $2,380(80)$ & $937(82)$ \\
\hline \multicolumn{4}{|l|}{ Mode of arrival } \\
\hline Ambulance/healthcare assisted & $14,071(24)$ & $1,084(36)$ & $445(39)$ \\
\hline Public/others ${ }^{\mathrm{b})}$ & $3,529(6)$ & 407 (14) & $136(12)$ \\
\hline Private vehicles & $42,260(71)$ & $1,485(50)$ & $573(50)$ \\
\hline \multicolumn{4}{|l|}{ Insurance } \\
\hline Commercial & $5,558(9)$ & $161(5)$ & $59(5)$ \\
\hline Government and charity & $27,811(46)$ & $1,884(63)$ & $712(62)$ \\
\hline Uninsured & $26,491(44)$ & $931(31)$ & $383(33)$ \\
\hline \multicolumn{4}{|l|}{ Triage ESI } \\
\hline ESI-1 & $481(0.8)$ & $19(0.6)$ & $10(0.9)$ \\
\hline $\mathrm{ESI}-2$ & $8,861(15)$ & $555(19)$ & $207(18)$ \\
\hline ESI-3 & $37,217(62)$ & $1,827(62)$ & $733(64)$ \\
\hline ESI-4 & $11,775(20)$ & $472(16)$ & $172(15)$ \\
\hline ESI-5 & $1,377(2)$ & $92(3)$ & $30(3)$ \\
\hline \multicolumn{4}{|l|}{ Primary care provider } \\
\hline Assigned & $22,930(38)$ & $1,314(44)$ & $511(44)$ \\
\hline Unassigned & $36,930(62)$ & $1,662(56)$ & $643(56)$ \\
\hline \multicolumn{4}{|l|}{ Homeless } \\
\hline No & $54,970(92)$ & $2,227(75)$ & $867(75)$ \\
\hline Yes & $4,890(8)$ & $749(25)$ & $287(25)$ \\
\hline \multicolumn{4}{|l|}{ History of alcohol use } \\
\hline No & $38,586(64)$ & $1,515(51)$ & $567(49)$ \\
\hline Yes & $21,274(36)$ & $1,461(49)$ & $587(51)$ \\
\hline \multicolumn{4}{|l|}{ History of substance use } \\
\hline No & $48,095(80)$ & $1,941(65)$ & $724(63)$ \\
\hline Yes & $11,765(20)$ & $1,035(35)$ & $430(37)$ \\
\hline \multicolumn{4}{|l|}{ Medication prescription upon discharge } \\
\hline No & $20,131(34)$ & $1,453(49)$ & $553(48)$ \\
\hline Yes & $39,729(66)$ & $1,523(51)$ & $601(52)$ \\
\hline \multicolumn{4}{|l|}{ EDRV } \\
\hline \multicolumn{4}{|l|}{ Return mode of arrival } \\
\hline Ambulance/healthcare assisted & & $1,055(36)$ & $427(37)$ \\
\hline Public/others ${ }^{b)}$ & & $436(15)$ & $167(15)$ \\
\hline Private vehicles & & $1,476(50)$ & $558(48)$ \\
\hline \multicolumn{4}{|l|}{ Return ESI } \\
\hline ESI-1 & & $30(1)$ & $14(1)$ \\
\hline $\mathrm{ESI}-2$ & & $574(19)$ & $253(22)$ \\
\hline ESI-3 & & $1,694(57)$ & $648(56)$ \\
\hline ESI-4 & & $526(18)$ & $189(16)$ \\
\hline ESI-5 & & $144(5)$ & $48(4)$ \\
\hline \multicolumn{4}{|l|}{ Return ED disposition } \\
\hline No significant disposition deviations & & $2,118(71)$ & $826(72)$ \\
\hline Uncertain disposition deviations & & $235(8)$ & $78(7)$ \\
\hline Significant disposition deviations & & $623(21)$ & $250(22)$ \\
\hline
\end{tabular}

Values are presented as number (\%).

EDRV, emergency department return visit; ED, emergency department; ESI, Emergency Severity Index.

a) Others refer to as Asian, American Indian or Alaska Native, Native Hawaiian or Pacific Islander, or unknown. ${ }^{b}$ Others refer to as public vehicle, ambulatory, and wheelchair. 
was selected based on Likelihood Ratio Tests, Akaike Information Criterion and Bayesian Information Criterion values. All analyses were performed using Stata ver. 14.2 (StataCorp., College Station, TX, USA) with P-value $<0.5$ considered statistically significant.

\section{RESULTS}

\section{General information}

A total of 63,990 patient encounters were enrolled in this study comprising 43,809 unique patients. A detailed interpretation of patient information is provided in Table 1. Two rounds of provider reviews were performed to determine whether EDRV were related to initial ED visits. Interrater agreement was 0.74 (first round, $P<0.05$ ) and 0.93 (second round, $P<0.05$ ), indicating a strong level of agreement between providers. Additional electronic health record reviews were stopped after the second round of reviews. Among all enrolled patient encounters, 6.45\% $(4,130)$ were 72 hours EDRV; 4.65\% $(2,976)$ were determined related EDRV and $1.80 \%(1,154)$ were considered unrelated. Briefly, return patient characteristics consisted primarily of male, geriatric, homeless patient populations with government/charity funded insurance. These patient encounters also had a higher incidence of history of alcohol or drug abuse and psychiatric conditions. Subsequently, all EDRV patients were included in a subgroup study. Analysis of this subgroup found 21\% (873) of patients had significant EDRVDD while $71 \%(2,944)$ had no disposition deviations (Table 1).

\section{Risks predictive of ED returns}

After adjusting for all potential risks, it was found that there were nine independent risks favoring EDRV: geriatrics; non-Caucasian
Independent risks

Age $\geq 65$

Sex (female vs. male)

Race (Caucasian vs. others)

Mode of arrival (health assist/public vs. private) Initial disposition (LWBS/AMA/eloped vs. discharge) Insurance (government vs. commercial) Homeless (yes vs. no) Prescription upon discharge (yes vs. no) History of alcohol abuse (yes vs. no) History of substance abuse (yes vs. no) Acute injury (yes vs. no) History of psychiatric conditions (yes vs. no)

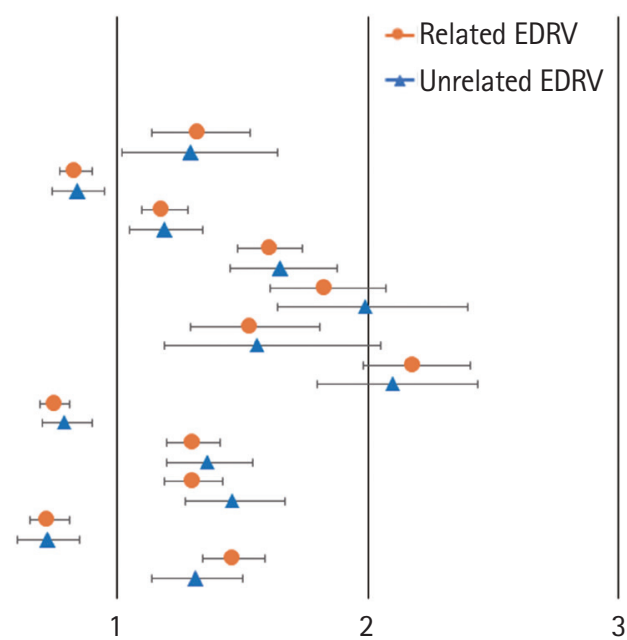

Adjusted odds ratios of risks predictive of EDRV

Fig. 1. Risks predictive of emergency department return visits (EDRVs) within 72 hours. LWBS, left without being seen; AMA, against medical advice.

Independent risks

Age $\geq 65$

Mode of arrival (public vs. private) Initial ED length of stay

Homeless (yes)

Primary care physician assigned (yes)

Return emergency severity index

$$
\begin{aligned}
& \text { - Related EDRV } \\
& \text { — Unrelated EDRV }
\end{aligned}
$$

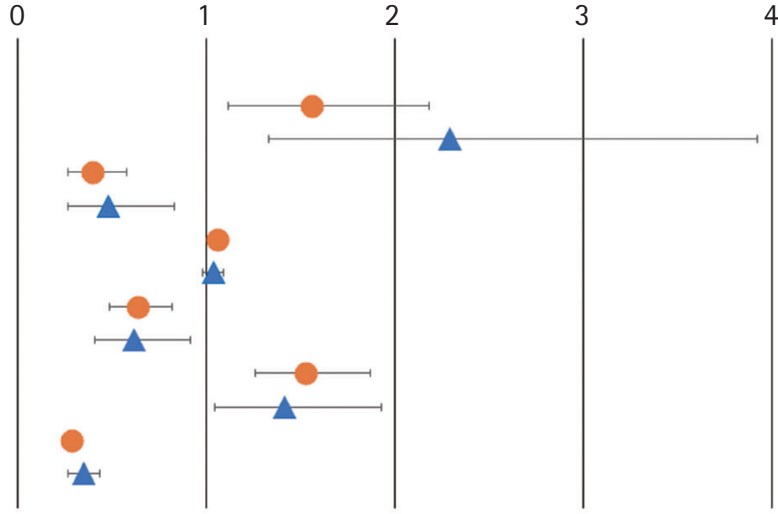

Adjusted odds ratios of risks predictive of ED disposition deviation

Fig. 2. Risks predictive of emergency department (ED) significant disposition deviations from initial visits. EDRV, emergency department return visit. 
races; homelessness; initial ED visit LWBS/AMA/eloped; history of alcohol or substance abuse; psychiatric conditions; patients with government/charity insurances; and mode of arrival to the ED other than private car or taxi (e.g., healthcare assisted or public transportation). Female patients with acute injuries and medications prescribed upon ED discharge were considered having relatively less risks for ED returns (Fig. 1 and Supplementary Table 1). More importantly, risks predictive of related and unrelated EDRV were very similar (Fig. 1).

\section{Risks predictive of significant ED return disposition deviations}

After adjusting for all potential confounders, the three independent risks that predicted more significant EDRVDD were: geriatric age, patients with primary care physician assigned, and longer ED length of stay during EDRV as compared to initial visit. Three risks predicted less significant deviations: mode of arrival to the ED (public or other transportation); homelessness; and patient triaged with lower level of acuity (Fig. 2 and Supplementary Table 2). Overall, these independent risks predicted significant EDRVDD regardless of its relation (related vs. unrelated) to the initial ED visits.

\section{DISCUSSION}

It is well known that risks predictive of EDRV are multifactorial and may vary among different patient populations. ${ }^{21,22}$ Our findings showed similar risks predictive of EDRV despite association (related versus unrelated) with the initial ED visits. This relationship factor has not been previously reported in the literature. ${ }^{14,21,23}$ Additionally, this study further emphasizes disposition deviations among EDRV patients and identifies different risk prediction models which are infrequently found in previous studies. More importantly, similar risk patterns are again revealed regarding the ED disposition deviation predictive model despite its link to initial ED visits. Based on our predictive models, though EDRV patients are a different patient population in comparison to patients with no returns, related and unrelated EDRV patients can be categorized into one group for future quality improvement interventions. Since all EDRV patients might be affected by the same risk patterns, we suggest that further differentiating EDRV patients with respect to related versus unrelated return visits might not be necessary.

Specific study methodology was used in this study. Stepwise sequential interrater agreement performed in this study, though subjective and time consuming, demonstrates less bias regarding determination of related versus unrelated EDRV, similar to the modified Delphi technique. ${ }^{24}$ Additionally, further identifying risks pre- dicting related versus unrelated EDRV and EDRVDD using a multivariate multinomial logistic regression model provided us an optimal analytic method to minimize confounding biases while allowing more appropriate outcome categorizations than a traditional multivariate logistic regression model with binary outcomes. ${ }^{20}$

We identified over $6 \%$ of patients experiencing 72 hours EDRV which is similar to those previously reported. ${ }^{5,8,14,25}$ Most EDRV predictive risks identified in this study have been reported in other studies demonstrating consistent findings. ${ }^{14,16,26-28}$ Among all risks predictive of EDRV, homelessness and patient LWBS/AMA/eloped dispositions were among the top two contributing factors. Many previous studies identified subgroups of patient populations (e.g., patients with high psychosocial risks) that tended to have higher EDRV whose general characteristics matched well with our top risks in this study. ${ }^{12,26,28-30}$ All potential independent risks also fit well into three major risk areas (patient, illness, and system/provider related) thus further confirming such risks being multifactorial.

Similar risk patterns were noted between related versus unrelated EDRV subgroups indicating that it is unnecessary to differentiate related and unrelated EDRV. It is well known that geriatric patients and patients with higher level acuity presentations tend to have higher frequency disposition deviations when comparing index to return visits. ${ }^{27}$ Geriatric patients have greater potential for severe disease progression despite subtle symptoms presentation at the initial ED visit. Patients with higher level acuity presentations usually indicate more emergent/severe disease patterns requiring further evaluation. ${ }^{21,27}$ This might explain how common ED provider behavior (e.g., universal high-risk potential consideration among all EDRV patients) influences EDRV patient dispositions although we are unable to provide direct evidence for such linkage in this study. Moreover, no significant management deviation was noted among non-geriatric, homeless patients with lower acuity level presentations.

Our findings indicate that EDRV patients are a special patient population and the risks predictive of EDRV and EDRVDD are multifactorial. Such findings provide some indirect evidence for future interventions in this special patient population such as, but not limited to, minimizing EDRV by applying out-of-hospital outreach programs or urgent primary care physician follow up for low acuity, homeless, or non-geriatric EDRV patients since their management is rarely altered. However, lacking direct evidence of such linkage, a prospective observational study with large sample size is warranted for external validation.

In summary, over 6\% of patients experienced EDRV within 72 hours of initial ED visit. Though risks predicting such revisits were multifactorial, similar risks were identified for both EDRV and EDRVDD regardless of whether EDRV was related versus unrelat- 
ed to initial visit. Therefore, it is reasonable to consider EDRV as a single entity in terms of patient care outcome measurements in future studies.

This is a single-center retrospective study which cannot demonstrate causality due to limited information accuracy and potential selection bias. Second, we enrolled all EDRV patients at the study ED but are unable to identify and enroll ones that might have visited other EDs within 72 hours of index visit to the study ED. Third, though high fidelity was observed between individual reviewers regarding determination of related versus unrelated EDRV, we were unable to completely rule out case-by-case inaccuracy due to lack of a gold standard. Furthermore, as risks predictive of EDRV or EDRVDD are multifactorial, we may have overlooked other potential risks not considered in this study. Fourth, homeless patients tended to have high psychosocial risks and their ED return pattern might be different than the general patient population. In this study, we did not investigate ED return and disposition deviation risks in detail among homeless patients which could generate potential patient selection bias. Finally, considering ED disposition deviations as a singular end-point may be short-sighted thereby excluding additional potential clinically relevant data that might be associated with an extended investigation incorporating inhospital morbidity, mortality, and/or short-term progress reports following hospital admission which might be linked to EDRV. Future studies should be carried out investigating more robust network data to include large regional and/or national samples analyzing extended outcomes.

\section{SUPPLEMENTARY MATERIAL}

Supplementary Tables are available from: https://doi.org/10.15441/ ceem.18.024.

\section{CONFLICT OF INTEREST}

No potential conflict of interest relevant to this article was reported.

\section{REFERENCES}

1. Lindsay $P$, Schull M, Bronskill $S$, Anderson G. The development of indicators to measure the quality of clinical care in emergency departments following a modified-delphi approach. Acad Emerg Med 2002;9:1131-9.

2. Duseja R, Bardach NS, Lin GA, et al. Revisit rates and associated costs after an emergency department encounter: a multistate analysis. Ann Intern Med 2015;162:750-6.
3. Depiero AD, Ochsenschlager DW, Chamberlain JM. Analysis of pediatric hospitalizations after emergency department release as a quality improvement tool. Ann Emerg Med 2002;39:15963.

4. Wang HY, Chew G, Kung CT, Chung KJ, Lee WH. The use of Charlson comorbidity index for patients revisiting the emergency department within 72 hours. Chang Gung Med J 2007; 30:437-44.

5. Akenroye AT, Thurm CW, Neuman Ml, et al. Prevalence and predictors of return visits to pediatric emergency departments. J Hosp Med 2014;9:779-87.

6. Liaw SJ, Bullard MJ, Hu PM, Chen JC, Liao HC. Rates and causes of emergency department revisits within 72 hours. J Formos Med Assoc 1999;98:422-5.

7. van der Linden $M C$, Lindeboom $R$, de Haan $R$, et al. Unscheduled return visits to a Dutch inner-city emergency department. Int J Emerg Med 2014;7:23.

8. Rising KL, Victor TW, Hollander JE, Carr BG. Patient returns to the emergency department: the time-to-return curve. Acad Emerg Med 2014;21:864-71.

9. Wu CL, Wang FT, Chiang YC, et al. Unplanned emergency department revisits within 72 hours to a secondary teaching referral hospital in Taiwan. J Emerg Med 2010;38:512-7.

10. Cheng SY, Wang HT, Lee CW, Tsai TC, Hung CW, Wu KH. The characteristics and prognostic predictors of unplanned hospital admission within 72 hours after ED discharge. Am J Emerg Med 2013;31:1490-4.

11. Imsuwan I. Characteristics of unscheduled emergency department return visit patients within 48 hours in Thammasat University Hospital. J Med Assoc Thai 2011;94 Suppl 7:S73-80.

12. Smith MW, Stocks C, Santora PB. Hospital readmission rates and emergency department visits for mental health and substance abuse conditions. Community Ment Health J 2015;51: 190-7.

13. Dy CJ, Lyman S, Do HT, Fabricant PD, Marx RG, Green DW. Socioeconomic factors are associated with frequency of repeat emergency department visits for pediatric closed fractures. J Pediatr Orthop 2014;34:548-51.

14. Ko M, Lee $Y$, Chen $C$, Chou P, Chu D. Incidence of and predictors for early return visits to the emergency department: a population-based survey. Medicine (Baltimore) 2015;94:e1770.

15. Ngai KM, Grudzen CR, Lee R, Tong VY, Richardson LD, Fernan$\operatorname{dez} A$. The association between limited English proficiency and unplanned emergency department revisit within 72 hours. Ann Emerg Med 2016;68:213-21.

16. Jacobstein CR, Alessandrini EA, Lavelle JM, Shaw KN. Unscheduled revisits to a pediatric emergency department: risk 
factors for children with fever or infection-related complaints. Pediatr Emerg Care 2005;21:816-21.

17. McCusker J, Ionescu-Ittu R, Ciampi A, et al. Hospital characteristics and emergency department care of older patients are associated with return visits. Acad Emerg Med 2007;14:42633.

18. Sung SF, Liu KE, Chen SC, Lo CL, Lin KC, Hu YH. Predicting factors and risk stratification for return visits to the emergency department within 72 hours in pediatric patients. Pediatr Emerg Care 2015;31:819-24.

19. Ali AB, Place R, Howell J, Malubay SM. Early pediatric emergency department return visits: a prospective patient-centric assessment. Clin Pediatr (Phila) 2012;51:651-8.

20. Van Hoorde K, Vergouwe Y, Timmerman D, Van Huffel S, Steyerberg EW, Van Calster B. Assessing calibration of multinomial risk prediction models. Stat Med 2014;33:2585-96.

21. Sauvin G, Freund Y, Saidi $K$, Riou B, Hausfater P. Unscheduled return visits to the emergency department: consequences for triage. Acad Emerg Med 2013;20:33-9.

22. Jin $B, Z$ hao $Y$, Hao $S$, et al. Prospective stratification of patients at risk for emergency department revisit: resource utilization and population management strategy implications. BMC Emerg Med 2016;16:10.

23. Ryan J, Hendler J, Bennett KP. Understanding emergency department 72-hour revisits among medicaid patients using electronic healthcare records. Big Data 2015;3:238-48.

24. Buetow SA, Coster GD. New Zealand and United Kingdom experiences with the RAND modified Delphi approach to producing angina and heart failure criteria for quality assessment in general practice. Qual Health Care 2000;9:222-31.

25. Goldman RD, Ong M, Macpherson A. Unscheduled return visits to the pediatric emergency department-one-year experience. Pediatr Emerg Care 2006;22:545-9.

26. Verelst S, Pierloot S, Desruelles D, Gillet JB, Bergs J. Shortterm unscheduled return visits of adult patients to the emergency department. J Emerg Med 2014;47:131-9.

27. Hu KW, Lu YH, Lin HJ, Guo HR, Foo NP. Unscheduled return visits with and without admission post emergency department discharge. J Emerg Med 2012;43:1110-8.

28. Geirsson OP, Gunnarsdottir OS, Baldursson J, Hrafnkelsson B, Rafnsson V. Risk of repeat visits, hospitalisation and death after uncompleted and completed visits to the emergency department: a prospective observation study. Emerg Med J 2013; 30:662-8.

29. Lam CN, Arora S, Menchine M. Increased 30-day emergency department revisits among homeless patients with mental health conditions. West J Emerg Med 2016;17:607-12.

30. Wang $H$, Nejtek VA, Zieger D, et al. The role of charity care and primary care physician assignment on ED use in homeless patients. Am J Emerg Med 2015;33:1006-11. 
Supplemental Table 1. Risk factors predictive of 72-hour emergency department return visits

\begin{tabular}{|c|c|c|c|}
\hline \multirow{2}{*}{ Independent risks } & Related return & No return & Unrelated return \\
\hline & Adjusted odds ratio (95\% Cl) & Reference & Adjusted odds ratio $(95 \% \mathrm{Cl})$ \\
\hline \multicolumn{4}{|l|}{ Age (yr) } \\
\hline Less than 64 & Reference & & Reference \\
\hline 65 or older & $1.32(1.14-1.53)$ & 1 & $1.29(1.02-1.64)$ \\
\hline \multicolumn{4}{|l|}{ Sex } \\
\hline Male & Reference & & Reference \\
\hline Female & $0.83(0.77-0.90)$ & 1 & $0.84(0.74-0.95)$ \\
\hline \multicolumn{4}{|l|}{ Race } \\
\hline White & Reference & & Reference \\
\hline Black/others ${ }^{\text {a) }}$ & $1.18(1.10-1.28)$ & 1 & $1.19(1.05-1.34)$ \\
\hline \multicolumn{4}{|l|}{ Mode of arrival } \\
\hline Private & Reference & & Reference \\
\hline Healthcare assisted/public/others ${ }^{b)}$ & $1.61(1.48-1.74)$ & 1 & $1.65(1.45-1.88)$ \\
\hline \multicolumn{4}{|l|}{ Initial ED disposition } \\
\hline Discharge & Reference & & Reference \\
\hline LWBS/AMA/eloped & $1.83(1.61-2.07)$ & 1 & $1.99(1.64-2.40)$ \\
\hline \multicolumn{4}{|l|}{ Insurance } \\
\hline Commercial & Reference & & Reference \\
\hline Government/charity & $1.53(1.29-1.81)$ & 1 & $1.56(1.19-2.05)$ \\
\hline Uninsured ${ }^{c)}$ & $1.08(0.91-1.29)$ & 1 & $1.20(0.91-1.58)$ \\
\hline \multicolumn{4}{|l|}{ Medication prescription upon discharge } \\
\hline No medication prescription & Reference & & Reference \\
\hline Medication prescription given & $0.75(0.69-0.81)$ & 1 & $0.79(0.70-0.90)$ \\
\hline \multicolumn{4}{|l|}{ Homeless } \\
\hline No & Reference & & Reference \\
\hline Yes & $2.18(1.98-2.41)$ & 1 & $2.10(1.80-2.44)$ \\
\hline \multicolumn{4}{|l|}{ Acute injury } \\
\hline No & Reference & & Reference \\
\hline Yes & $0.72(0.65-0.81)$ & 1 & $0.72(0.60-0.85)$ \\
\hline \multicolumn{4}{|l|}{ History of alcohol abuse } \\
\hline No & Reference & & Reference \\
\hline Yes & $1.30(1.20-1.41)$ & 1 & $1.36(1.20-1.54)$ \\
\hline \multicolumn{4}{|l|}{ History of substance abuse } \\
\hline No & Reference & & Reference \\
\hline Yes & $1.30(1.19-1.42)$ & 1 & $1.46(1.27-1.67)$ \\
\hline \multicolumn{4}{|l|}{ History of psychiatric conditions } \\
\hline No & Reference & & Reference \\
\hline Yes & $1.46(1.34-1.59)$ & 1 & $1.31(1.14-1.50)$ \\
\hline
\end{tabular}

$\mathrm{Cl}$, confidence interval; ED, emergency department; LWBS, left without being seen; AMA, against medical advice.

${ }^{\text {a) }}$ Others (race): American Indian, Alaska Native, Asian, Native Hawaiian and Pacific Islanders, Patient refused to provide, or unknown. ${ }^{b}$ Others (mode of arrival): ambulatory, police, wheelchair, or unknown. ${ }^{c}$ Not included in Fig. 1 due to no statistical significance at $\mathrm{a}=0.05$. 
Supplementary Table 2. Risk factors predictive of ED significant disposition deviations

\begin{tabular}{|c|c|c|c|}
\hline \multirow[t]{2}{*}{ Independent risks } & $\begin{array}{l}\text { Significant disposition deviation from } \\
\text { related ED returns }(n=2,976)\end{array}$ & $\begin{array}{c}\text { No significant } \\
\text { disposition deviation }\end{array}$ & $\begin{array}{l}\text { Significant disposition deviation from } \\
\text { unrelated ED returns }(n=1,154)\end{array}$ \\
\hline & Adjusted odds ratio (95\% Cl) & Reference & Odds ratio $(95 \% \mathrm{Cl})$ \\
\hline \multicolumn{4}{|l|}{ Age (yr) } \\
\hline Younger than 65 & Reference & & Reference \\
\hline 65 or older & $1.56(1.12-2.18)$ & 1 & $2.29(1.33-3.92)$ \\
\hline \multicolumn{4}{|l|}{ Return mode of arrival } \\
\hline Private & Reference & & Reference \\
\hline Ambulance/healthcare assistant ${ }^{a)}$ & $1.03(0.83-1.27)$ & 1 & $0.88(0.63-1.23)$ \\
\hline Public/others ${ }^{b)}$ & $0.40(0.27-0.58)$ & 1 & $0.48(0.27-0.83)$ \\
\hline Initial ED length of stay & $1.06(1.03-1.09)$ & 1 & $1.04(0.98-1.09)$ \\
\hline Return Emergency Severity Index & $0.29(0.24-0.34)$ & 1 & $0.35(0.27-0.44)$ \\
\hline \multicolumn{4}{|l|}{ Homeless } \\
\hline No & Reference & & Reference \\
\hline Yes & $0.64(0.49-0.82)$ & 1 & $0.62(0.41-0.92)$ \\
\hline \multicolumn{4}{|l|}{ Primary care physician assigned } \\
\hline No & Reference & & Reference \\
\hline Yes & $1.53(1.26-1.87)$ & 1 & $1.42(1.05-1.93)$ \\
\hline
\end{tabular}

$\mathrm{ED}$, emergency department; $\mathrm{Cl}$, confidence interval.

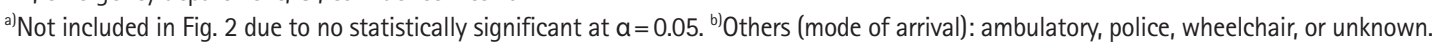

